\title{
A Novel Morphometric Nomenclature to Evaluate Osseointegration of Intraosseous Implants
}

\author{
DOI: $10.17691 / \mathrm{stm} 2018.10 .3 .1$
}

Received December 26, 2017

A.V. Volkov, PhD, Senior Researcher, Laboratory for Studies of the Connective Tissue ${ }^{1}$;

B.S. Smbatyan, MD, DSc, Dental Surgeon²;

D.N. Nazaryan, MD, PhD, Senior Researcher, Head of the Department of Maxillofacial and Plastic Surgery ${ }^{3}$;

A.A. Muraev, MD, PhD, Associate Professor, Department of Maxillofacial Surgery and Implantology4; Associate Professor, Department of Maxillofacial Surgery and Surgical Dentistry ${ }^{5}$

${ }^{1}$ N.N. Priorov National Medical Research Center of Traumatology and Orthopaedics, Healthcare Ministry of Russia, 10 Priorov St., Moscow, 127299, Russia;

2“Dention” Dental Clinic, 15 Rustaveli St., Moscow, 127254, Russia;

${ }^{3}$ The Russian National Research Center of Surgery named after B.V. Petrovsky, Moscow, 2 Abrikosovsky per., Moscow, 119991, Russia;

${ }^{4}$ Privolzhsky Research Medical University, 10/1 Minin and Pozharsky Square, Nizhny Novgorod, 603005, Russia;

${ }^{5}$ Peoples' Friendship University of Russia, 6 Miklukho-Maklaya St., Moscow, 117198, Russia

The aim of the study was to develop an optimal system of criteria to evaluate the osseointegration of intraosseous dental implants and then to analysis and predict their static and dynamic competence.

Results. We propose a unified nomenclature for the morphometric evaluation of osseointegration of dental implants; this novel system is based on the recommendations of the American Society for Bone and Mineral Research as well as recent studies on cranial and maxillofacial implants including our own experience.

Conclusion. The proposed nomenclature can be used as a basic unified platform for experimental and clinical studies on the efficacy and safety of dental implants.

Key words: osseointegration; intraosseous implants; histomorphometry; maxillofacial surgery.

\section{Introduction}

A dental implant (DI) inherently represents a foreign body even if it is made of bioinert alloys using improved methods of surface treatment; therefore, studies on osseointegration - the process of DI engraftment remains relevant. Today, it is almost impossible to evaluate the efficacy and safety of intraosseous Dls without analyzing their relationship with the recipient bone tissue [1]. In addition, a DI that carries the tooth's function is subjected to axial, lateral, rotational and combined loads during chewing, which has its own impact on the bone tissue. Understanding the relationships on the "implant-bone" border is important for predicting the "survival" of Dls, for developing optimal materials, and designing the implant surface [2].
It should be noted that titanium implants have long ceased to be used only for the elimination of dental defects; extraoral or cranial implants are able to replace lost ears, finger phalanxes, the orbit, the upper jaw, and the nose [3] - all those are widely used in the current traumatology and orthopedics [4].

The increasing use of intraosseous implants necessitates a comprehensive assessment of the safety and efficacy of the implantation procedure. Histomorphometric analysis is conventionally considered the major approach to the problem. Although the current histomorphological technique lacks standardized parameters for evaluating the osseointegration of implants, the main criteria are well known. For example, the main index of osseointegration is the BIC (boneimplant contact), which characterizes the contact

Corresponding author: Alexey V. Volkov, e-mail: alex.volkoff@gmail.com 
between the implant surface and the bone; or the interface of the bone matrix of the recipient bed. The value of this parameter depends on the time elapsed after implantation, the initial state of the bone, the load on the implant, and the surface and shape of the implant itself. The interface between the connective tissue and the bone marrow is an important index as well. Other indices of direct interaction between the implant and body tissues are optional and used sporadically. Thus, in some reports, more attention is paid to the contact between the implant surface and the bone tissue (both maternal and newly formed), in others - to the contact between the implant and granulation tissue. Each group of researchers offers its own criteria depending on specific tasks [5-7].

Recently, an attempt was made to reconcile the approaches pursued by morphologists and clinicians [8]. In the present study, we undertake to systematize the experience accumulated in the area of cranial and maxillofacial surgery including our own results and reports by others.

The aim of the study was to develop an optimal system of criteria suitable for the evaluation of osseointegration of intraosseous dental implants and then to analysis and predict their static and dynamic competence.

The proposed standard is based on the international system of bone tissue evaluation, developed by the American Society for Bone and Mineral Research (ASBMR) in the previous and updated version [9, 10].

Stereometric assessment of interaction between orthopedic devices and the bone tissue; orientation of the dental implant section. Morphologists studying

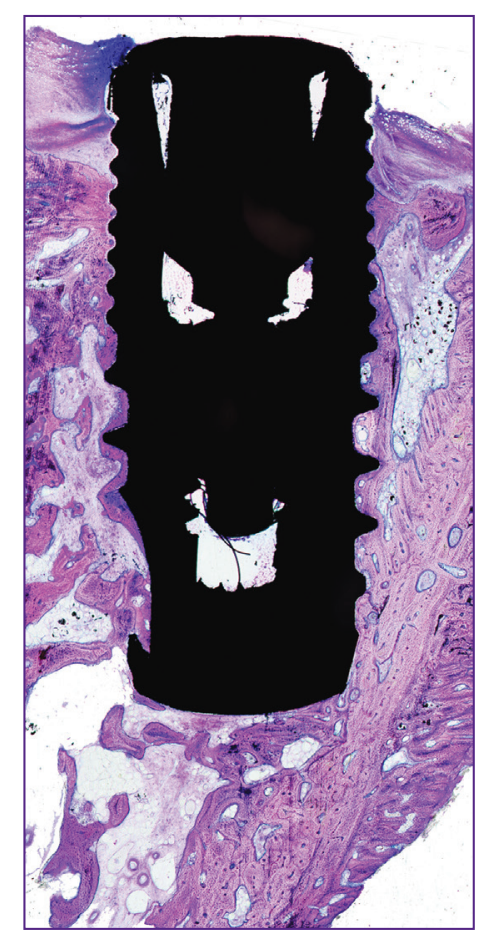

Figure 1. A longitudinal section of a Conmet implant; staining with toluidine blue and acidic fuchsine; $\times 50$

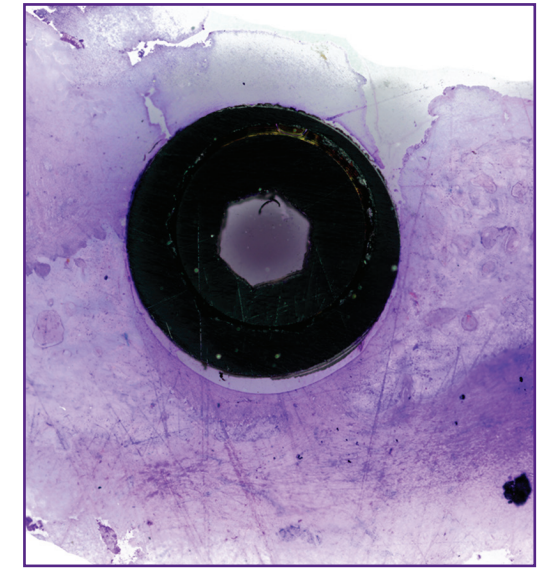

Figure 2. A transverse section of an implant; made to assess its interaction with the soft tissues of the alveolar ridge; staining with sky-blue trichrome; $\times 50$

the osseointegration and DI survival may face problems concerning not only the implant interaction with bone structures but also the interaction between the implant's neck (or its abutment) and the soft tissues of the alveolar ridge. This information is needed to predict the possibility of cervical peri-implantitis and subsequent cervical bone resorption. Traditionally, the osseointegration is assessed using sagittal (longitudinal) sections (Figure 1). This is due to the inability to perform thin serial sections of implants and the difficulty to examine the surrounding tissues. In such cases, we used sections made in the frontal plane; those showed morphological details of the soft tissue attached to the implant's neck (Figure 2).

A stereometric system for assessing the osseointegration of dental implants. To characterize the interaction between an implant and the recipient bone bed, the following parameters were selected:

the primary non-load osseointegration - the interaction between the DI and the bone during its engraftment within a two-stage clinical protocol including the suturing of flaps over the implant - to assess the biocompatibility of the implant, its alloy and surface design;

the impact of the DI on surrounding tissues when it functions under a load after the onset of primary osseointegration;

the osseointegration and/or the impact of the implant on the surrounding bone and soft tissues immediately after its installation and under a functional load.

Parameters characterizing the process of osseointegration. In the literature, there are no unified recommendations for determining the engraftment and survival of DIs. Most authors adapt the Branemark experience and use the BIC index, which characterizes the contact between the implant outer surface and the bone tissue interface [5]. This parameter does not discern the bone tissue by maturity and the time of formation. Later, other parameters, presented in the 
The unified system of indices used to assess the osseointegration and the interaction between dental implants (based on reports [1, 2, 4, 5, 7, and 11] and authors' own results)

\begin{tabular}{|c|c|c|c|}
\hline Currently used terminology & Proposed terminology & Abbreviation & Units \\
\hline \multicolumn{4}{|c|}{ Parameters describing the interaction between tissues and implant interface } \\
\hline O, old bone, BIC & Old bone-implant contact & $\mathrm{OBIC}$ & $\%$ \\
\hline N, new bone, BIC & New bone-implant contact & $\mathrm{nBIC}$ & $\%$ \\
\hline Marrow spaces & Marrow implant contact & MalC & $\%$ \\
\hline FIC, provisional matrix & Fibrous implant contact & $\mathrm{FIC}$ & $\%$ \\
\hline Particles & Material implant contact & MatlC & $\%$ \\
\hline Clot free from inflammatory infiltrate & Fibrin implant contact & FilC & $\%$ \\
\hline Inflammatory infiltrate & Inflammatory infiltrate implant contact & IIIC & $\%$ \\
\hline- & Tooth implant contact & TolC & $\%$ \\
\hline \multirow[t]{2}{*}{ Other tissues } & Gingival implant contact & GglC & $\%$ \\
\hline & Epithelial implant contact & EplC & $\%$ \\
\hline \multicolumn{4}{|c|}{ Parameters describing the implant-associated osteogenesis and resorption } \\
\hline- & Osteoblast-populated surface & I.Ob.S & $\mu \mathrm{m}$ \\
\hline- & Osteoid-covered surface & I.OS & $\mu \mathrm{m}$ \\
\hline- & Number of osteoblasts on the implant surface & N.I.Ob & piece \\
\hline- & Depth of peri-cervical resorption & $\mathrm{dRB}$ & $\mathrm{mm}$ \\
\hline- & Width of peri-cervical resorption & wRB & $\mathrm{mm}$ \\
\hline \multicolumn{4}{|c|}{ Parameters describing the bone tissue under a load } \\
\hline- & $\begin{array}{l}\text { Thickness of the cortical plate } \\
\text { of the implant-contacting bone }\end{array}$ & I.Ct.Th & $\mu \mathrm{m}$ \\
\hline- & $\begin{array}{l}\text { Thickness of the trabeculae } \\
\text { in the implant-contacting spongy bone }\end{array}$ & I.Tb.Th & $\mu \mathrm{m}$ \\
\hline- & $\begin{array}{l}\text { Deviation angle of trabeculae } \\
\text { of spongy bone }\end{array}$ & IsB & degree \\
\hline
\end{tabular}

Table, were added to evaluate the interaction. Upon analyzing the literature for the use of the BIC index, we found no unified terminology describing the engraftment and survival of the implant.

Based on the international experience, we propose an original unified system of indices for the stereometric and morphometric evaluation that is adjustable to the tasks faced by the morphologists (see the Table).

Parameters of interaction between the tissues and the implant interface. Interaction of biological tissues with a DI is the crucial factor reflecting the efficacy and safety of the implantation procedure. Thus, the ability of the implant to get integrated, i.e. to directly interact with the bone tissue, is denoted as BIC. In turn, if the surface of the implant is osteoconductive and it shows osteoid deposits or osteoblast proliferation, the BIC abbreviation is added with the prefix "n" (new). The term will then look "nBIC" (new bone-implant contact), which means "newly formed bone on the implant surface". To define the "old" (maternal) bone of the recipient bed, one should use the prefix "o" (old), i.e. "oBIC" (old boneimplant contact) (Figure 3).

At different stages of osseointegration, for example,

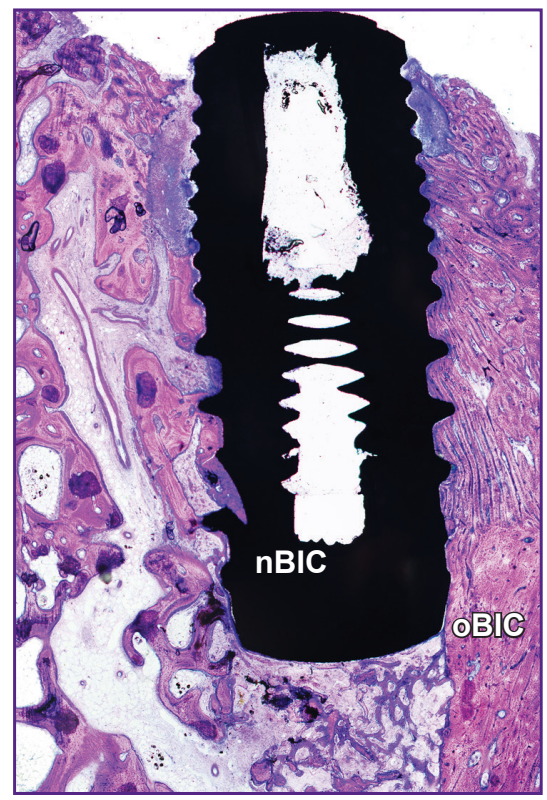

Figure 3. Newly formed (nBIC) and maternal (oBIC) bones in contact with the implant; staining with toluidine blue and acidic fuchsine; $\times 50$ 


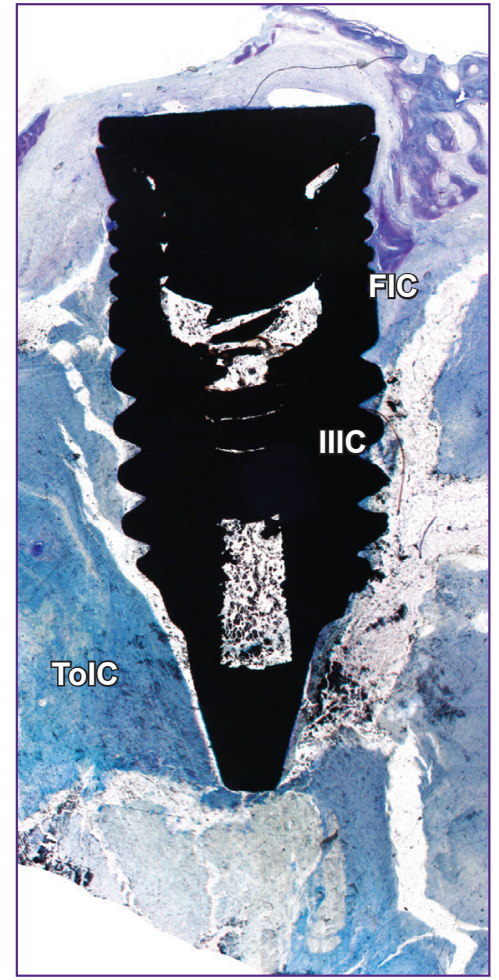

Figure 4. A transverse section of an implant; made at the point bordering a tooth (TolC), an inflammatory infiltrate (IIIC), and a fibrous tissue (FIC); staining with toluidine blue; $\times 50$

in the case of low osteoconductivity, the DI surface may contact the bone marrow, granulation tissue, inflammatory infiltrate, or fibrous tissue. In these cases, the appropriate terms are designated in accordance with the recommendations of the ASBMR $[9,10]$ or their modified versions [8]: bone marrow - MalC (marrow implant contact); fibrous tissue - FIC (fibrous implant contact); inflammatory infiltrate - IIIC (inflammatory infiltrate implant contact); fibrin - FilC (fibrin implant contact); granules of osteoplastic material - MatlC (material implant contact); tooth (tooth root) - TolC (tooth implant contact) (Figure 4). In the cases of implant interaction with soft tissues, for example periodontium, subcutaneous tissue, exo-implants are named as GglC (gingival implant contact) for periodontal tissue and EpIC (epithelial implant contact) for the skin, subcutaneous and other epithelial tissues.

The interaction of Dls with tissues is expressed as a ratio $(\%)$ of their surface contacting the tissues to the entire surface of the implant.

Parameters of osteogenesis and resorption associated with the implant. The currently produced dental, auricular and other implants have their surface modified, namely, it is coated with substances that ensure the best compatibility with the surrounding tissues. There are additional ways of the surface modification, such as increasing the contact area or impregnating the surface with bioactive molecules (for example, calcium salts). In these cases, the implant becomes not just a mechanical prosthesis, but a bioprosthesis possessing additional qualities.

During the DI engraftment, the modified surface can attract some bone tissue and result in the formation of osteoid deposits or multiple proliferating osteoblasts. In other cases of poor biocompatibility, inflammatory cells can be found on the surface of the implant.

In comparative studies on the modified surface, it is advisable to indicate the area occupied by the osteoid I.OS and/or osteoblasts - I.Ob.S. It would be also useful to count the number of the osteoblastic cellular elements on the implant surface - N.I.Ob.

In some cases, in order to assess the bone resorption around the implant neck, it is necessary to determine the depth and width of the bone loss. In this case, the terms $\mathrm{dRB}$ (depth resorption bone) and wRB (width resorption bone) are recommended.

Parameters of the bone tissue under a load. Any study of $\mathrm{DI}$ osseointegration must include the determination of changes in the surrounding tissues under a load on the implant. The load and the impact of external forces on the implant cause the adjacent bone tissue to get restructured depending on the DI design. The load vectors are directed according to the laws of mechanics (resistance of materials) and the theory of threaded joints [11, 12].

Bone structures associated with the implant. The result of the primary osseointegration of an intraosseous $\mathrm{DI}$ is the formation of lamellar bone tissue that contacts the outer surface of the implant. This bone structure, which has a histological and functional similarity to the cortical plate and inter-root septum of the alveolar part of the jaw, undergoes a number of dynamic changes, which depend on the load amplitude and the load vectors. Implant-associated bone trabeculae adjoin the bone plate located around the implant; the trabeculae are formed as a specific response to the load and they are able to dynamically change their thickness.

During the osseointegration process, a non-loaded, primarily stabilized, implant is subjected to the formation of specific bone structures; the similar process occurs to a prosthetic implant under a load, in case the implant is biocompatible. In those bone structures, the lamellar bone tissue around the implant is biomechanically related to the cortical plate of the jaw ridge through the cervical angle, as well as to the implant surface, and also to the implant-associated bone trabeculae.

Thus, to describe the characteristics of a DI under a mechanical load, one should look into the tissue surrounding the implant at a distance of up to $0.5 \mathrm{~cm}$ from the highest point of the maximally protruding thread (if any) for both dental and exo-implants. The respective area in orthopedic implants can be much larger.

The distribution of the load vectors determines the rearrangement of both the cortical bone plate around the implant and the trabeculae of the spongy bone. 
Thus, vertical axis loading leads to the formation of bone trabeculae at an angle of $45-65^{\circ}$ of the implant axis. This phenomenon is commonly denoted as the IsB index. Combined with this index, the thickness of the bone cortical plate contacting the implant - I.Ct.Th, and the

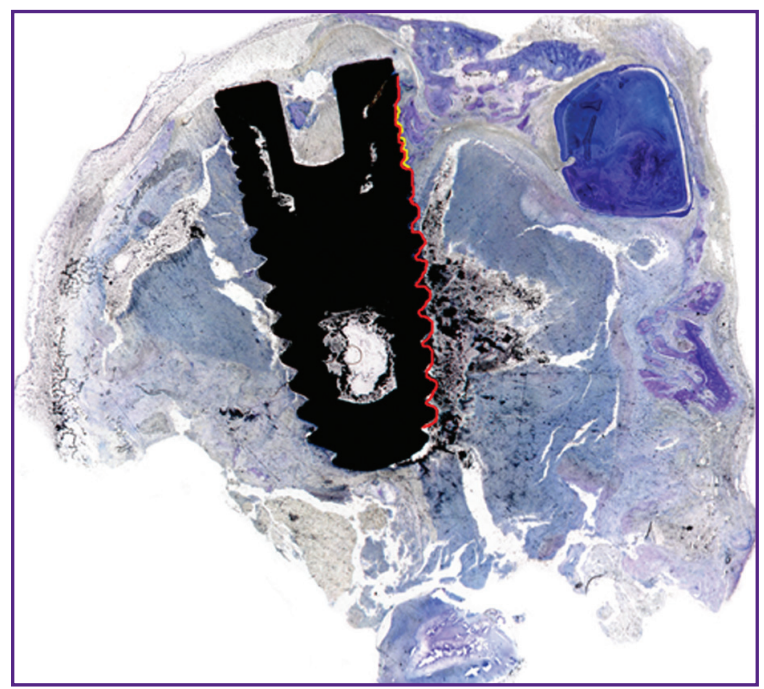

Figure 5. Measurement of the area of contact between an implant and the bone tissue (one of the stages)

Red line - the total area of contact; yellow line - the area of contact with the newly formed bone tissue (nBIC) thickness of the trabeculae in the implant - associated spongy bone - I.Tb.Th, are to be determined.

Measurements to assess the osseointegration. In order to study the process of osseointegration, the tools to measure straight and curved lines, angles, and also to count points are required (Figures 5, 6).

The relative area of tissue contact with the intraosseous implant can be calculated from the structural characteristics of the implant. The part of the implant designed for contacting the bone must be measured in its entirety, for any type of the contacting tissue. In the case of cervical resorption, some of the implant threads can be stripped off the bone tissue; consequently, the free space is filled with a fibrous or granulation tissue. Those elements, which were not intended to be placed inside the bone (abutment) are not included in the assessment of osseointegration. If part of the implant penetrates under the mucous membrane of the maxillary sinus, it is recommended not to evaluate this sample because of the high probability of an artifact.

Determining the structure of the implantassociated bone tissue. In our previous studies, we described the determination of the basic indicators of bone tissue around the implant [8]. When the bone and its structural components change, one should take into account the reasonable distances between the implants, between the implants and the neighboring teeth or other anatomical structures that bear the load. Most often, such changes are found within $1 \mathrm{~cm}$ from the DI.

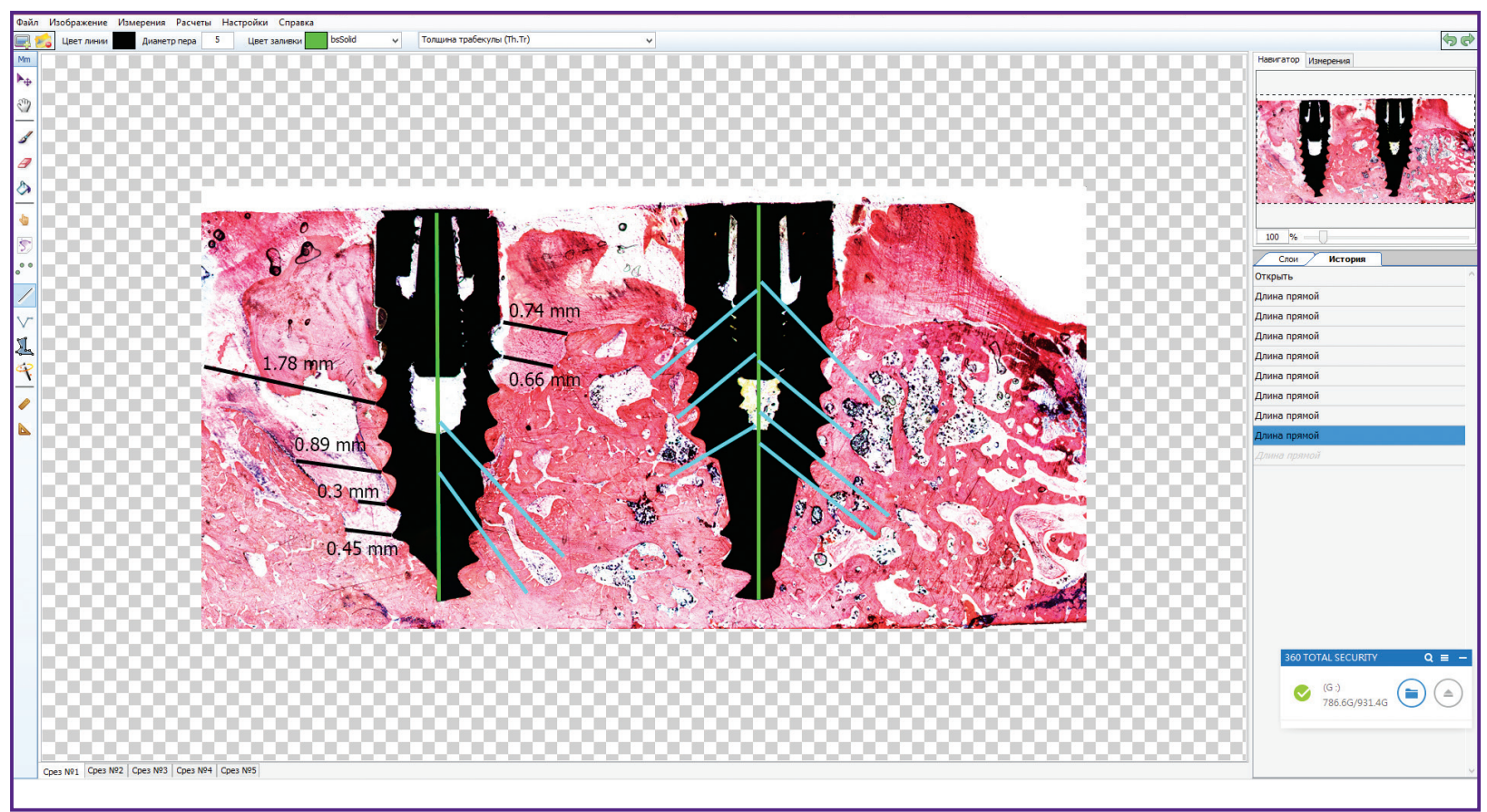

Figure 6. A window of the MegaMorph12 morphometric program (Russia)

Determination of the interaction between an implant and surrounding tissues; blue lines - the measurement of the deviation angles between the implant-associated trabeculae and the central axis (green lines) of the implant; black lines - determination of the resorption around the implant 


\section{Conclusion}

We propose an original morphometric platform to characterize the interaction between dental implants and the recipient bed; the present methodology is designed to facilitate the determination of the efficacy and safety of the implant. The proposed system is not the absolute standard; rather it can serve as a basis for creating an algorithm for evaluating the process of osseointegration. Additional parameters can be sensibly added to this system, for example, elements of the ASBMR nomenclature, both original and modified. The presented morphometric platform can also be used in experimental and clinical studies on endoprostheses, exoprostheses, and other orthopedic devices.

Acknowledgments. The authors are privileged to propose the name "Karen" for the bone plate formed around the dental implant, in honor and commemoration of Karen Levonovich Gabrielyan, a talented scientist who contributed a lot in the field of maxillofacial surgery.

Financial support. The study was not funded by any sources.

Conflict of interest. The authors confirm the absence of conflicts of interest that could influence their work.

\section{References}

1. Froum S.J., Simon H., Cho S.C., Elian N., Rohrer M.D., Tarnow D.P. Histologic evaluation of bone-implant contact of immediately loaded transitional implants after 6 to 27 months. Int J Oral Maxillofac Implants 2005; 20(1): 54-60.

2. Dimitriou R., Babis G.C. Biomaterial osseointegration enhancement with biophysical stimulation. J Musculoskelet Neuronal Interact 2007; 7(3): 253-265.

3. Thiele O.C., Brom J., Dunsche A., Ehrenfeld M., Federspil P., Frerich B., Hölzle F., Klein M., Kreppel M., Kübler A.C., Kübler N.R., Kunkel M., Kuttenberger J., Lauer G., Mayer B., Mohr C., Neff A., Rasse M., Reich R.H., Reinert S., Rothamel D., Sader R., Schliephake H., Schmelzeisen R., Schramm A., Sieg P., Terheyden H., Wiltfang J., Ziegler C.M.,
Mischkowski R.A., Zöller J.E. The current state of facial prosthetics - a multicenter analysis. J Craniomaxillofac Surg 2015; 43(7): 1038-1041, https://doi.org/10.1016/j. jcms.2015.04.024.

4. Ochsner P.E. Osteointegration of orthopaedic devices. Semin Immunopathol 2011; 33(3): 245-156, https://doi. org/10.1007/s00281-011-0241-4.

5. Behfarnia P., Khorasani M.M., Birang R., Abbas F.M. Histological and histomorphometric analysis of animal experimental dehiscence defect treated with three bio absorbable GTR collagen membrane. Dent Res J (Isfahan) 2012; 9(5): 574-581, https://doi.org/10.4103/1735-3327. 104876.

6. Gulati M., Anand V., Govila V., Jain N., Rastogi P., Bahuguna R., Anand B. Periodontio-integrated implants: a revolutionary concept. Dent Res J (Isfahan) 2014; 11(2): 154-162.

7. Favero V., Lang N.P., Rossi F., Favero R., Baffone D., Botticelli D. Peri-implant tissues morphometry at SLActive surfaces. An experimental study in the dog. Clin Oral Implants Res 2016; 27(8): 993-998, https://doi.org/10.1111/clr.12719.

8. Volkov A.V., Badalyan V.A., Kulakov A.A., Babichenko I.I., Kapanadze G.D., Stankova N.V. Histomorphological researches of relationship of a bone with dental implant. Biomeditsina 2012; 4: 96-100.

9. Dempster D.W., Compston J.E., Drezner M.K., Glorieux F.H., Kanis J.A., Malluche H., Meunier P.J., Ott S.M., Recker R.R., Parfitt A.M. Standardized nomenclature, symbols, and units for bone histomorphometry: a 2012 update of the report of the ASBMR Histomorphometry Nomenclature Committee. J Bone Miner Res 2013; 28(1): 2-17, https://doi. org/10.1002/jbmr.1805.

10. Parfitt A.M. Bone histomorphometry: proposed system for standardization of nomenclature, symbols, and units. Calcif Tissue Int 1988; 42(5): 284-286, https://doi.org/10.1007/ bf02556360.

11. Natali A.N., Pavan P.G., Ruggero A.L. Analysis of bone-implant interaction phenomena by using a numerical approach. Clin Oral Implants Res 2006; 17(1): 67-74, https:// doi.org/10.1111/j.1600-0501.2005.01162.x.

12. Birger I.A., losilevich G.B. Rez'bovye $i$ flantsevye soedineniya [Threaded and flange connection]. Moscow: Mashinostroenie; 1990. 